\title{
Increased soil moisture aggravated the competitive effects of the invasive tree Rhus typhina on the native tree Cotinus coggygria
}

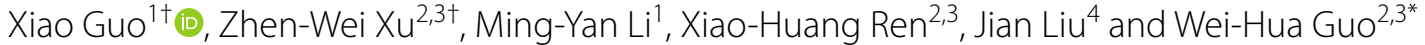

\begin{abstract}
Background: Invasive exotic species have caused significant problems, and the effects of extreme precipitation and drought, which might occur more frequently under the global climate change scenarios, on interspecific relationship between invasive and native species remain unclear.

Results: We conducted a greenhouse experiment with three soil water levels (30-40\%, 50-60\%, and 70-80\% of field capacity) and two cultivation treatments (monoculture pots, one seedling of either species and mixture pots, one seedling of each species) to investigate soil water content effects on the relationship between invasive Rhus typhina and native Cotinus coggygria. Rhus typhina had lower height but bigger crown area than C. coggygria in the monoculture treatment. Rhus typhina had higher height, bigger crown area and total biomass than C. coggygria in the mixture treatment. Drought decreased the growth parameters, total chlorophyll concentration, and leaf biomass, but did not change gas exchange and other biomass parameters in R. typhina. The growth parameters, leaf area index, biomass parameters, total chlorophyll concentration, and net photosynthetic rate of $C$. coggygria decreased under drought conditions. The log response ratio (InRR), calculated as In (total biomass of a target plant grown in monoculture/total biomass of a target plant grown in mixed culture), of $R$. typhina was lower than that of $C$. coggygria. The InRR of $R$. typhina and C. coggygria decreased and increased with increase in soil water content, respectively.

Conclusions: Rhus typhina has greater capacity to relatively stable growth to the drought condition than C. coggygria and has strong competition advantages in the mixture with C. coggygria, especially in the drought condition. Our study will help understand the causes of invasiveness and wide distribution of $R$. typhina under various moisture conditions and predict its expansion under climate change scenarios.
\end{abstract}

Keywords: Competition, Biomass allocation, Photosynthesis, Plant invasion, Water gradient

\section{Background}

The global climate change has resulted in an increasing trend in extreme precipitation in some areas, and severe drought is predicted to occur more frequently in the future in several regions of the world [1]. Water limitation is one of the main factors limiting plant growth and

\footnotetext{
*Correspondence: guo_wh@yahoo.com

${ }^{\dagger}$ Xiao Guo and Zhen-Wei Xu should be considered as joint first authors

${ }^{2}$ Institute of Ecology and Biodiversity, College of Life Sciences, Shandong

University, Qingdao 266237, People's Republic of China

Full list of author information is available at the end of the article
}

is the most general type of stress experienced by plants [2]. Drought restrains eco-physiological performances of plants, such as photosynthesis and primary productivity $[3,4]$ and changes the community structure [5], creating an opportunity for the invasion of exotic species, most likely leading to in the decline of biodiversity [6] and instability of the entire ecosystem $[7,8]$.

Identifying the determinants of the invasiveness of alien plant species is essential in invasion ecology, and it has considerable implications [9]. It has been reported that the invasive species always have faster growth rate,

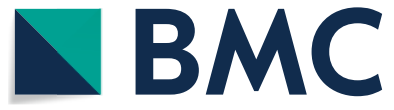

(C) The Author(s) 2020. This article is licensed under a Creative Commons Attribution 4.0 International License, which permits use, sharing, adaptation, distribution and reproduction in any medium or format, as long as you give appropriate credit to the original author(s) and the source, provide a link to the Creative Commons licence, and indicate if changes were made. The images or other third party material in this article are included in the article's Creative Commons licence, unless indicated otherwise in a credit line to the material. If material is not included in the article's Creative Commons licence and your intended use is not permitted by statutory regulation or exceeds the permitted use, you will need to obtain permission directly from the copyright holder. To view a copy of this licence, visit http://creativeco mmons.org/licenses/by/4.0/. The Creative Commons Public Domain Dedication waiver (http://creativecommons.org/publicdomain/ zero/1.0/) applies to the data made available in this article, unless otherwise stated in a credit line to the data. 
stronger photosynthesis [10], and more plastic biomass allocation [11] than those of native species [12]. These differences can be explained by the phenotypic divergence hypothesis $[13,14]$, which suggests that the higher the difference in functional traits between the invasive and native species, the higher the probability of successful invasion. However, based on the process of habitat filtering, phenotypic convergence hypothesis $[15,16]$ states the importance of environmental factors and holds that invasive and coexisting native species have a strong similarity in functional traits. This strong similarity confers the exotic species the ability to adapt to local environments and to invade native communities more easily $[15,17]$. The phenotypic convergence hypothesis has been supported by some empirical studies $[18,19]$. Despite numerous studies, there is no consensus on this hypothesis.

The competition for resources is a crucial process that influences plant competitive interactions and invasiveness $[6,9,20]$. According to the theory of fluctuating resource availability [21], increased resources will increase probability of invasion success of invaders into a plant community. In addition, native species have been reported to outperform the invasive species under limited resource conditions [17]. However, it is not always the case as invasive species were more or similar efficient to acquire resources compared to native species under conditions of low resources [22-26]. Invasive plant can change the leaf traits to adapt the shade environment, such as expanding leaf area to increase light acquirement [27]. Besides, if the growth of exotic and native species is restricted by different resources, the increase in certain resources might not exacerbate the competitive effects of exotics [28, 29]. Therefore, the consistent conclusion is still unclear.

Water plays an important role in the growth and physiology of plants and significantly affects the interaction between native and invasive species [30]. Drier soils have been demonstrated to significantly increase the total biomass of invasive species, whereas, the native species are unaffected by the soil water status [31]. Invasive plant can adjust the resource allocation to leaf, which can decrease leaf are to reduce transpiration loss [32]. However, the relative growth rate of invasive Lantana camara decreased under drought condition; thus, limiting its invasion into arid and sub-arid environments [33]. Furthermore, some studies found that native species is a better competitor than invasive species, regardless of the water regime [34] or water supply pattern [35]. Experimental evidence is still insufficient and the effect of soil moisture on the competitive effects of invasive species remains unclear.
To examine the effects of invasive species on native species in response to various water conditions, we conducted a greenhouse experiment with an invasive species (Rhus typhina) and a confamiliar native species (Cotinus coggygria). Rhus typhina, a large shrub or small tree native to eastern North America [36], is highly invasive in Europe [37], and it is also regarded as an invader in China [38]. The species can propagate through root suckers to occupy the territory rapidly, and it can tolerate extremely poor soils with rapid reproduction and vigorous growth [39]. Cotinus coggygria is widely distributed in the warmtemperate zone of China by local adaptation [40], and it tends to be a small tree or a shrub $[41,42]$. Both the species are regarded as ornamental plants, and they are known for their red autumnal leaves [39, 42]. R. typhina and C. coggygria exist in the temperate deciduous forest in the eastern Asia and have strong competition with each other, especially at the stage and have similar ecological niche at the stage of seedlings and mature trees. Besides, the two species are widely used for reforestation in North China [39].

Most of previous studies have compared mixture treatment containing two species in one pot with monoculture treatment containing two or more single-species plants in one pot $[36,43-45]$. This kind of experimental design might underestimate the effects of interspecific competition, because the comparison is made between interspecific and intraspecific competition, with intraspecific competition as control $[31,36,46]$. Therefore, in the present study, we set up a monoculture treatment with one species per pot as the control to avoid the interference of intraspecific competition. We addressed the following questions:

1. Is the invasive $R$. typhina a superior competitor in mixtures? Does $R$. typhina show convergence or divergence in functional traits relative to $C . \operatorname{cog}$ gygria?

2. What functional traits confer the competitive advantage to the superior competitor?

3. Does water availability affect the competition dominance between R. typhina and C. coggygria?

\section{Results}

\section{Plant growth}

In the monoculture pots, the shoot height and RGR-H of $R$. typhina were lower than those of C. coggygria, whereas the crown area of $R$. typhina was higher than that of $C$. coggygria (Fig. 1). In the mixed culture pots, the shoot height and crown area of $R$. typhina were higher than those of C. coggygria (Fig. 1). All the growth parameters, including the shoot height, crown area, and RGR-H of $R$. 


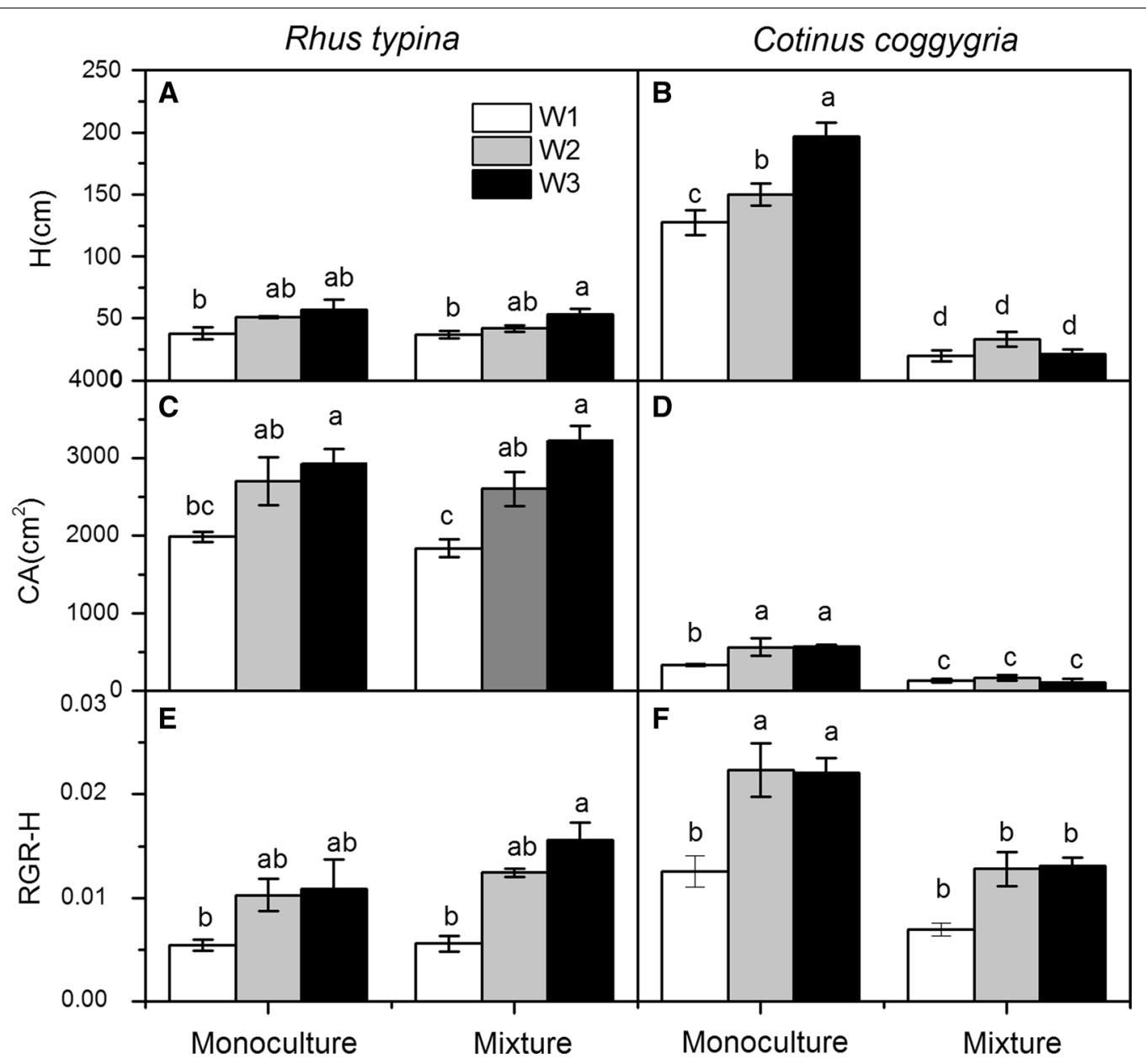

Fig. 1 Height $(H ; \mathbf{A}, \mathbf{B})$, crown area $(C A ; C, D)$, and relative growth rate for shoot height (RGR-H; E, F) of Rhus typhina and Cotinus coggygria at the end of the experiment under three water and two cultivation regimes. Data in the figure are mean $\pm S E(n=5)$. Different letters denote significant differences $(p \leq 0.05)$ with Duncan's method

typhina were generally decreased under drought condition, but not by cultivation (Table 1; Fig. 1). The interaction of drought and cultivation had no effect on any of the growth parameters of $R$. typhina (Table 1). All the growth parameters of C. coggygria in the monoculture pots generally decreased with decreasing soil moisture content, whereas, those of C. coggygria in the mixture pots were similar across all water treatments (Fig. 1).

\section{Leaf traits}

In the monoculture pots, the LAI of $R$. typhina was higher than that of C. coggygria (Fig. 2). In the mixture pots, the SLA of R. typhina was lower than that of C. coggygria (Fig. 2). Water had no effect on the SLA and LAI of the two species (Table 1). Furthermore, the SLA of $R$. typhina was not affected by cultivation treatment as well. The SLA and LAI of C. coggygria generally increased in the mixture pots, compared with those of C. coggygria in the monoculture pots (Table 1; Fig. 2B, D). On the contrary, the LAI of $R$. typhina was decreased by mixed culture (Table 1, Fig. 2A).

\section{Biomass and allocation}

In the monoculture pots, the total biomass and leaf biomass of R. typhina were higher than those of C. coggygria under W1 (30-40\% of field capacity) and W2 (50-60\% of field capacity) conditions, but lower than those of $C$. coggygria under W3 (70-80\% of field capacity) condition. The root biomass of R. typhina was higher than that of $C$. coggygria in all the water treatments. In the mixture pots, the total biomass, leaf biomass, stem biomass, and root biomass of R. typhina were higher than those of C. $\operatorname{cog}$ gygria in all the water treatments. 
Table 1 Results of two-way ANOVA for the effects of water treatments, plant cultivation and their interactions on performance and functional traits of Rhus typhina and Cotinus coggygria

\begin{tabular}{|c|c|c|c|c|c|c|c|c|c|c|c|c|}
\hline & \multicolumn{6}{|c|}{ Rhus typhina } & \multicolumn{6}{|c|}{ Cotinus coggygria } \\
\hline & \multicolumn{2}{|c|}{ Water treatments } & \multicolumn{2}{|c|}{ Plant cultivation } & \multicolumn{2}{|c|}{$\begin{array}{l}\text { Interaction } \\
\text { effects }\end{array}$} & \multicolumn{2}{|c|}{ Water treatments } & \multicolumn{2}{|c|}{ Plant cultivation } & \multicolumn{2}{|c|}{$\begin{array}{l}\text { Interaction } \\
\text { effects }\end{array}$} \\
\hline & $\mathrm{F}$ & $p$ & $F$ & $p$ & $\mathbf{F}$ & $p$ & $\mathrm{~F}$ & $p$ & $F$ & $p$ & $\mathrm{~F}$ & $p$ \\
\hline Height $(\mathrm{cm})$ & 11.026 & $0.00^{* *}$ & 2.176 & 0.158 & 0.640 & 0.539 & 9.796 & $0.001^{* *}$ & 451.81 & $0.00^{* *}$ & 12.927 & $0.00^{* *}$ \\
\hline Crown area $\left(\mathrm{cm}^{2}\right)$ & 14.652 & $0.00^{* *}$ & 0.006 & 0.938 & 0.623 & 0.548 & 2.56 & 0.105 & 57.593 & $0.00^{* *}$ & 2.491 & 0.111 \\
\hline RGR-H & 12.186 & $0.000^{* *}$ & 3.314 & 0.084 & 0.994 & 0.388 & 14.483 & $0.000^{* *}$ & 34.468 & $0.000^{* *}$ & 0.832 & 0.450 \\
\hline $\mathrm{SLA}\left(\mathrm{cm}^{2} / \mathrm{g}\right)$ & 0.706 & 0.508 & 1.872 & 0.189 & 0.916 & 0.419 & 1.635 & 0.221 & 25.320 & $0.000^{* *}$ & 3.169 & 0.065 \\
\hline LAI & 0.076 & 0.927 & 90.586 & $0.000^{* *}$ & 1.478 & 0.262 & 3.317 & 0.061 & 6.311 & $0.022^{*}$ & 0.803 & 0.464 \\
\hline Chl & 4.824 & $0.029^{*}$ & 4.806 & $0.049^{*}$ & 0.023 & 0.977 & 0.242 & 0.788 & 6.182 & $0.027^{*}$ & 1.389 & 0.284 \\
\hline Chla/Chlb & 1.575 & 0.250 & 69.498 & $0.000^{* *}$ & 4.471 & $0.038^{*}$ & 1.653 & 0.229 & 3.024 & 0.106 & 7.301 & $0.007^{* *}$ \\
\hline $\mathrm{A}\left(\mu \mathrm{mol} \mathrm{m} \mathrm{m}^{-2} \mathrm{~s}^{-1}\right)$ & 0.161 & 0.853 & 0.011 & 0.918 & 2.133 & 0.161 & 3.010 & 0.087 & 36.548 & $0.000^{* *}$ & 0.924 & 0.424 \\
\hline$E\left(\mathrm{mmol} \mathrm{m} \mathrm{m}^{-2} \mathrm{~s}^{-1}\right)$ & 0.095 & 0.910 & 0.570 & 0.465 & 3.449 & 0.066 & 3.978 & $0.047^{*}$ & 9.718 & $0.009^{* *}$ & 0.111 & 0.896 \\
\hline Gs $\left(\mathrm{mmol} \mathrm{m} \mathrm{m}^{-2} \mathrm{~s}^{-1}\right)$ & 0.330 & 0.726 & 0.085 & 0.775 & 1.402 & 0.284 & 3.345 & 0.070 & 26.780 & $0.000^{* *}$ & 0.322 & 0.731 \\
\hline $\mathrm{Ci}\left(\mathrm{mmol} \mathrm{mol}{ }^{-1}\right)$ & 0.243 & 0.788 & 0.501 & 0.492 & 0.667 & 0.531 & 0.554 & 0.589 & 3.196 & 0.099 & 0.189 & 0.830 \\
\hline WUE & 0.438 & 0.655 & 5.854 & $0.032^{*}$ & 6.946 & $0.01^{*}$ & 1.714 & 0.221 & 12.42 & $0.004^{*}$ & 2.482 & 0.125 \\
\hline Total biomass (g) & 1.095 & 0.355 & 0.205 & 0.655 & 1.531 & 0.242 & 26.759 & $0.000^{* *}$ & 424.422 & $0.000^{* *}$ & 26.484 & $0.000^{* *}$ \\
\hline Leaf biomass (g) & 7.636 & $0.004^{* *}$ & 0.446 & 0.512 & 2.252 & 0.133 & 13.846 & $0.000^{* *}$ & 299.123 & $0.000^{* *}$ & 12.491 & $0.000^{* *}$ \\
\hline Stem biomass (g) & 0.517 & 0.605 & 0.003 & 0.958 & 1.213 & 0.319 & 19.317 & $0.000^{* *}$ & 535.269 & $0.000^{* *}$ & 19.453 & $0.000^{* *}$ \\
\hline Root biomass (g) & 1.708 & 0.208 & 1.655 & 0.214 & 1.903 & 0.176 & 4.100 & $0.034^{*}$ & 201.801 & $0.000^{* *}$ & 3.927 & $0.038^{*}$ \\
\hline Root-shoot ratio & 2.555 & 0.104 & 2.997 & 0.100 & 1.468 & 0.255 & 2.015 & 0.162 & 4.179 & 0.056 & 0.554 & 0.584 \\
\hline Leaf biomass ratio & 2.398 & 0.118 & 0.100 & 0.755 & 0.136 & 0.874 & 1.039 & 0.374 & 26.198 & $0.000^{* *}$ & 3.302 & 0.060 \\
\hline Stem biomass ratio & 0.371 & 0.695 & 1.584 & 0.223 & 0.770 & 0.477 & 2.447 & 0.115 & 127.233 & $0.000^{* *}$ & 3.689 & $0.045^{*}$ \\
\hline Root biomass ratio & 1.815 & 0.190 & 3.074 & 0.096 & 1.685 & 0.212 & 2.030 & 0.160 & 4.622 & $0.045^{*}$ & 0.544 & 0.590 \\
\hline
\end{tabular}

$R G R-H$ relative growth rate of height, $L A /$ leaf area index, SLA specific leaf area, $C h /$ total chlorophyll concentration, Chla/Chlb chlorophyll a to b ratio, $A$ maximum net photosynthetic rate, $E$ transpiration rate, Gs stomatal conductance, $C i$ Intercellular carbon dioxide concentration, WUE water use efficiency

Significant effects are indicated by italic and asterisks: ${ }^{*} p<0.01,{ }^{*} 0.01<p \leq 0.05$

In $R$. typhina, neither cultivation nor interaction between cultivation and water affected any of the biomass and allocation parameters and water affected only the leaf biomass (Table 1). The biomass parameters of $C$. coggygria were affected by cultivation and water, as well as their interaction. Mixed culture decreased all the biomass parameters and the SBR of C. coggygria; the LBR and RBR increased in the mixture pots compared with those in the monoculture pots (Table 1, Fig. 3). Drought generally decreased the leaf biomass of $R$. typhina in both the cultivation treatments (Table 1; Fig. 3C). Drought decreased all the biomass parameters of $C$. coggygria in the monoculture pots, whereas, those of C. coggygria in the mixture pots were not affected (Fig. 3).

\section{Chlorophyll and photosynthetic characters}

In the pots, the total chlorophyll concentration, A, and Gs of $R$. typhina was lower than those of C. coggygria (Fig. 4). In the mixture pots, the total chlorophyll concentration of $R$. typhina was lower than that of C. coggygria under W3 condition but higher than that under W1 and
W2 conditions (Fig. 4). In the mixture pots, the A and Gs of $R$. typhina were higher than that of $C$. coggygria under W1 and W2 conditions but were similar to those of $C$. coggygria under W3 condition. In the monoculture pots, the WUE of $R$. typhina was similar to that $C$. coggygria but was higher than that of $C$. coggygria in all the water treatments in mixture pots (Fig. 4). The WUE of R. typhina increased in the mixed culture compared to that in the monoculture, whereas the WUE of $C$. coggygria in the mixed culture decreased compared to that in the monoculture (Fig. 4).

The total chlorophyll concentration of R. typhina was decreased by increasing soil water content and mixed culture (Table 1, Fig. 4). The total chlorophyll concentration of C. coggygria was decreased by mixed culture (Table 1, Fig. 4). The chlorophyll a to chlorophyll b ratio of $R$. typhina was decreased by mixed culture (Table 1 , Fig. 4). The interaction between mixed culture and water affected the chlorophyll a to chlorophyll $b$ ratio in R. typhina (Table 1). However, neither mixed culture nor water had effect on the chlorophyll a to chlorophyll 


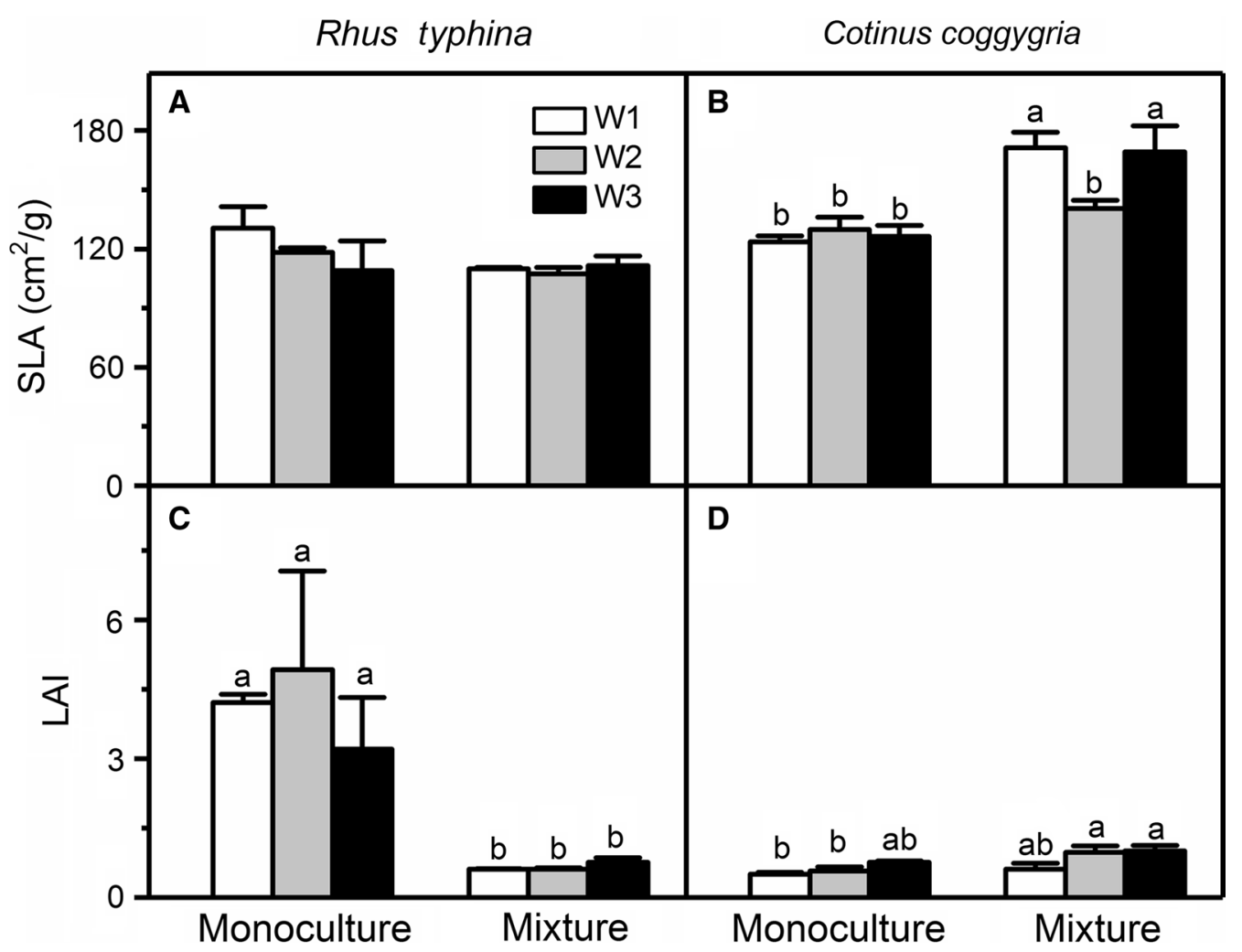

Fig. 2 Specific leaf area $(\mathbf{A}, \mathbf{B})$ and leaf area index $(L A \mid$; C, D) of Rhus typhina and Cotinus coggygria under three water and two cultivation regimes. Data in the figure are mean $\pm \operatorname{SE}(n=5)$. Different letters denote significant differences $(p \leq 0.05)$ with Duncan's method

b ratio of C. coggygria (Table 1). Plant cultivation and soil water content had no effect on the A of R. typhina, whereas mixed culture decreased that of C. coggygria (Table 1; Fig. 4). Mixed culture increased the WUE of R. typhina, but decreased that of C. coggygria (Table 1; Fig. 4).

\section{Competitive interaction between two species}

The $\ln R \mathrm{R}$ of $R$. typhina decreased with increase in soil moisture content, whereas, that of C. coggygria increased (Fig. 5). The $\ln R R$ of $R$. typhina was lower than that of C. coggygria (Fig. 5). In the W3 condition, the $\ln R R$ of $R$. typhina was less than zero, but the $\ln R R$ of C. coggygria was more than zero. In the W2 and W1 conditions, the $\ln R \mathrm{R}$ of both $R$. typhina and $C$. coggygria was more than zero and the $\ln R \mathrm{R}$ of $R$. typhina much larger than that of C. coggygria (Fig. 5).

\section{Discussion}

We conducted the phylogenetic comparison to minimize phylogenetic bias as trait differences among species strongly depend on phylogenetic relationships, growth forms and life forms $[9,22,47]$. $R$. typhina has high total biomass than C. coggygia in the mixture treatment. This indicates the strong competitive effects of $R$. typhina on $C$. coggygria and that $R$. typhina is the superior competitor. The $\ln R R$ further validated this observation as the $\ln R \mathrm{R}$ of $R$. typhina was always lower than that of $C$. $\operatorname{cog}$ gygria, indicating the competitive effects on $R$. typhina was less than that on $C$. coggygria. However, we cannot conclude that mixed culture with $C$. coggygria facilitated the growth of $R$. typhina in well-watered conditions even though the lnRR of $R$. typhina was below zero under W3 condition. This is because the total biomass of $R$. typhina remained unchanged across different water regimes. Rhus typhina has also been reported to outcompete native Quercus acutissima [36] and Vitex negundo [35].

\section{Interaction between R. typhina and C. coggygria under mixed culture condition}

The shifts in biomass allocation pattern of the introduced species can create a advantage for a species over their neighbors in response to different environmental conditions $[17,48]$. Both $R$. typhina and C. coggygria had high biomass in the W3 treatment but the total biomass of C. coggygria rapidly decreased with the soil moisture 


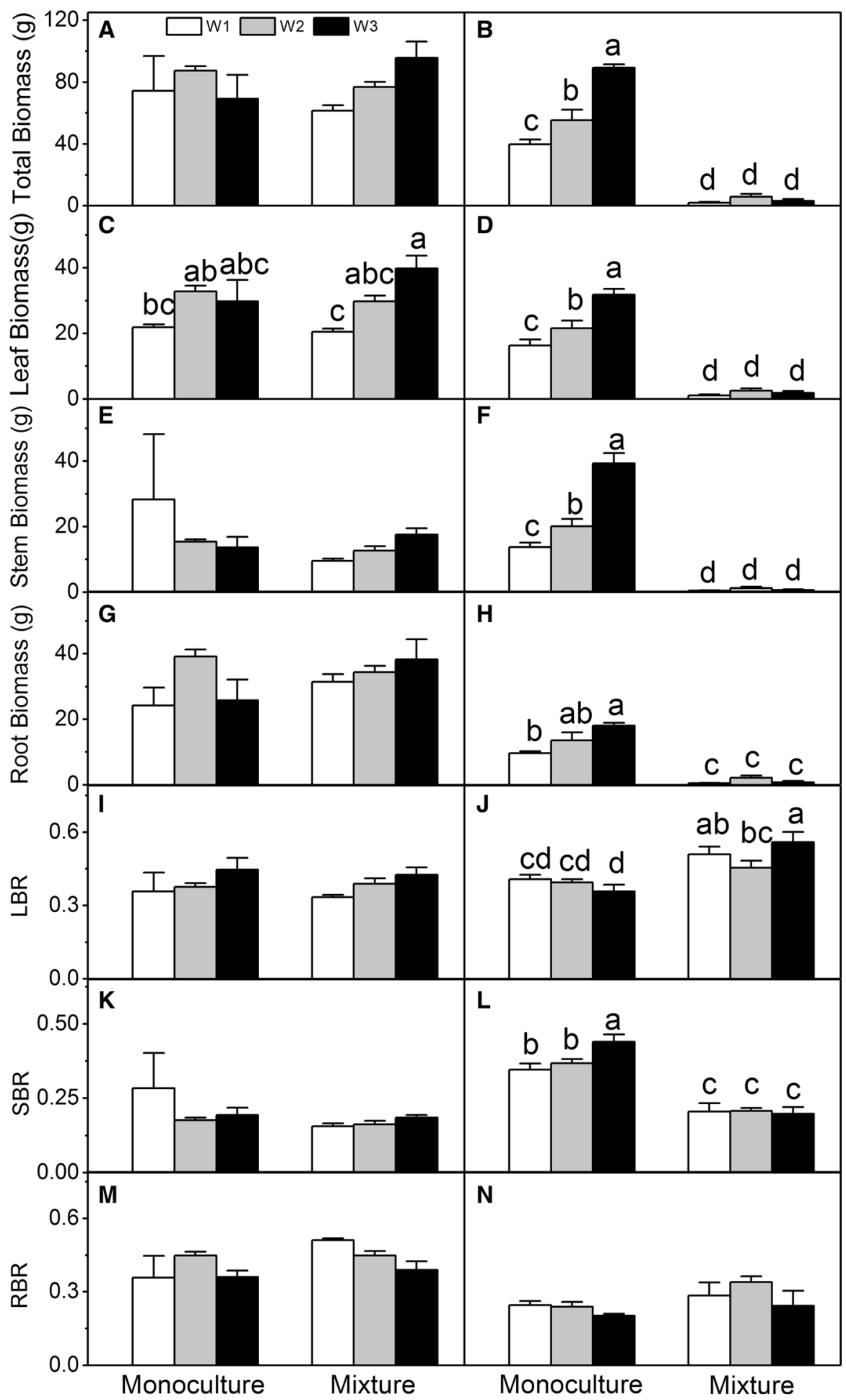

Fig. 3 Biomass parameters and biomass allocation parameters of Rhus typhina and Cotinus coggygria at the end of the experiment under three water and two cultivation regimes. The biomass parameters include total biomass $(\mathbf{A}, \mathbf{B})$, leaf biomass $(\mathbf{C}, \mathbf{D})$, stem biomass $(\mathbf{E}, \mathbf{F})$, and root biomass $(\mathbf{G}, \mathbf{H})$. The biomass allocation parameters include leaf biomass ratio $(L B R ; \mathbf{I}, \mathbf{J})$, stem biomass ratio $(S B R ; \mathbf{K}, \mathbf{L})$ and root biomass ratio (RBR; $\mathbf{M}, \mathbf{N})$. Data in the figure are mean $\pm S E(n=5)$. Different letters denote significant differences $(p \leq 0.05)$ with Duncan's method 


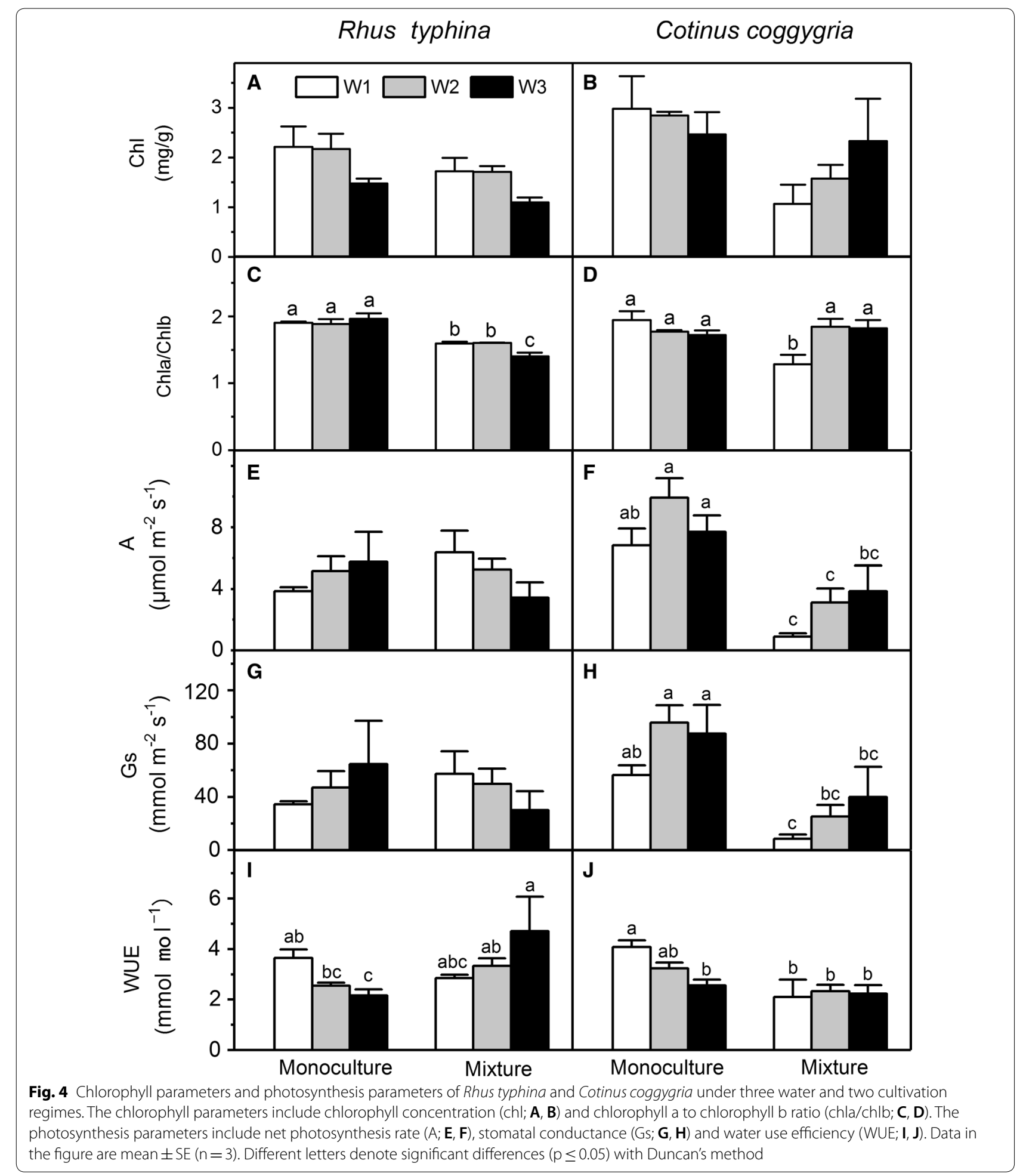

decreasing (Fig. 3). It indicated that $R$. typhina have stronger ability of adapting the drought environment than $C$. coggygria. Previous study found that $R$. typhina have higher total biomass facing both interspecific and intraspecific pressure than native plant under both drought and wet conditions [35]. Drought may be the disturbance for plant growth and invasive plant always can adapt to disturbance condition, based on the disturbance 


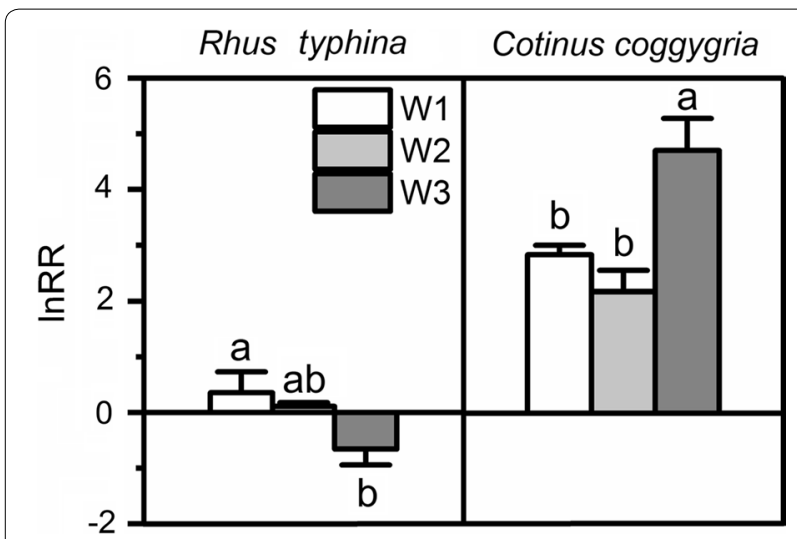

Fig. 5 The log response ratio (InRR) of Rhus typhina and Cotinus coggygria under three water regimes. The InRR was calculated as In (total biomass of a target plant grown in monoculture/total biomass of a target plant grown in mixed culture). Data in the figure are mean $\pm S E(n=5)$. Different letters denote significant differences $(p \leq 0.05)$ with Duncan's method

hypothesis [49]. Drought decreased the resource allocation of $C$. coggygria to stem but had no influence on the SBR of $R$. typhina (Fig. 3), and the R. typhina had lower height than C. coggygria (Fig. 1). R. typhina may have stronger the wood density and increase drought tolerance. Previous study found invasive tree Acacia mearnsii have higher wood density and resistive capacity to drought-induced cavitation than native trees in the introduced habitat [50]. Drought changes the species composition of native plant community and make more susceptible to invasion [51].

Shoot height and allocation to growth have been shown to promote invasiveness [47]. Higher shoot height, crown area, and leaf biomass in the present study together conferred $R$. typhina a competitive advantage over C. coggygria to obtain more light in mixture pots. According to the productivity-dependent scaling hypothesis, high aboveground productivity of the neighboring plant will impose strong asymmetric competition on the target plant $[52,53]$. This asymmetric competition for light has the strongest effect on the growth of small trees [52], i.e., C. coggygria seedlings in the present study, as the largest share of a vital resource can be gained by a plant through a slight height advantage over its neighbors [54]. The growth rate is probably a key functional trait linked to the invasiveness of a tree species, and it is highly associated with the invasion success of tree species $[9,47,55]$. In the mixture pots, $R$. typhina had slightly higher RGR-H and thus taller shoot to cover $C$. coggygria rapidly, suppressing the growth of the native species by shading it $[14,29]$. Besides, $R$. typhina enabled to inhibit the growth of shoot adjacent native plant Vitex negundo var. heterophylla in the interspecific competition although native plant had higher height than $R$. typhina facing intraspecific competition [35].

For C. coggygria, the RBR increased but the root biomass decreased in mixed culture, indicating that its ability to obtain water and potential nutrients was decreased by the strong competition from $R$. typhina. Larger root biomass of $R$. typhina than that of native species were found previously $[35,36]$. Furthermore, the RBR, root biomass, and WUE of $R$. typhina were higher than those of $C$. coggygria in mixed pots, which conferred $R$. typhina a conspicuous advantage to absorb more water and nutrients, and utilize the water more efficiently, hindering the growth of $C$. coggygria by decreasing its resource supply [29]. In this situation, C. coggygria invests more on biomass production to the root and leaf to cope with the intrusion of $R$. typhina, but this alteration in biomass allocation strategy still cannot offset the strong competitive effects from $R$. typhina. Considering the aboveground advantages mentioned above, we conclude that invasive alien species possess stronger light-capturing and water/nutrients-absorbing abilities, and water use efficiency than the native species by exhibiting more acquisitive functional traits. Previous found that facing the pressure of intraspecific competition, the $R$. typhina have higher total biomass and higher root biomass ratio to increasing the absorption nutrient than native plant Quercus acutissima Carr. and this advantage is also maintained during interspecies competition [36].

This conferred $R$. typhina an assimilatory advantage over C. coggygria; thus, contributing to higher performance than that of $C$. coggygria in the mixture pot. Higher photosynthetic traits are considered typical features for the success of invasive species [9, 14, 36, 47] and may be the reason for the higher growth rate of shoot height of $R$. typhina in the present study. Cotinus coggygria seedlings tend to grow upward (higher shoot height, RGR-H, and SBR than those of $R$. typhina) to reach the upper space with higher maximum net photosynthetic rate (A) than that of R. typhina. Rhus typhina seedlings tend to grow horizontally (higher crown area than that of $C$. coggygria) to capture more light and deeply to obtain more water and nutrient (higher root biomass and RBR than those of C. coggygria). Rhus typhina maintained larger crown area, leaf biomass, and root biomass than those of $C$. coggygria. The upward growth of $C$. coggygria seedlings was strongly inhibited (indicated by distinctly lower shoot height than that of C. coggygria in monoculture) and its A was decreased most likely due to the shade environment caused by the exceedingly larger canopy of $R$. typhina seedlings in the upper layer in the mixture. Eventually the total biomass of C. coggygria seedlings was extremely lower than that of $R$. typhina seedlings in the mixture pots. Therefore, 
the invasive $R$. typhina and the native $C$. coggygria displayed differences in most crucial functional parameters related to growth when they were cultivated separately or together, indicating that our results support the phenotypic divergence hypothesis [13] rather than the phenotypic convergence hypothesis $[15,16]$.

Invasive alien $R$. typhina possesses three advantages over the native $C$. coggygria in the mixed culture. First, with the stronger photosynthetic capacity than $C$. coggygria, $R$. typhina was able to achieve a higher growth rate and larger amount of carbohydrate production. Secondly, in the mixture pots, higher shoot height, crown area, RGR-H, and leaf biomass enabled R. typhina to exhibit dominance earlier by obtaining more light resource and shading the native $C$. coggygria. This asymmetric competition caused by the higher aboveground productivity of $R$. typhina over $C$. coggygria results in positive-feedback [53] to $R$. typhina, leading to even higher competitive advantages of $R$. typhina. Finally, the higher root biomass and WUE of R. typhina than C. coggygria conferred $R$. typhina the ability to obtain water and nutrients, and utilize water more efficiently.

\section{Drought relieves the competitive effects of R. typhina on $C$. coggygria}

Rhus typhina maintained its dominance under drought condition despite the fact that the competitive ability of $R$. typhina over C. coggygria decreased and concomitantly the competitive ability of $C$. coggygria over $R$. typhina increased to some extent. This observation generally supports the fluctuating resource availability theory [21], which holds that a plant community's invasibility decreases under limited resource conditions. The decrease in the competitive ability of $R$. typhina over $C$. coggygria under drought conditions was probably related to the growth inhibition of $R$. typhina (i.e., decrease in the shoot height, crown area, RGR-H, and leaf biomass) under drought conditions. However, the shoot height, crown area, RGR-H, and leaf biomass of $R$. typhina were still substantially higher than those of $C$. coggygria under drought condition, consistently conferring $R$. typhina an aboveground competitive advantage. Therefore, the asymmetric competition of $R$. typhina over $C$. coggygria was alleviated to some extent, because the light-capturing ability of $R$. typhina decreased to some extent, although this ability was still conspicuously stronger than that of C. coggygria. Rhus typhina has been shown to outperform native Vitex negundo under variable levels of water supply frequency but with a constant level of total supplied water [35].

Drought decreased all the biomass parameters of $C$. coggygria in the monoculture pots, whereas, those in the mixture pots was unaffected by drought, suggesting that the mixed culture alleviated the negative effects of drought on $C$. coggygria. This might be because $R$. typhina has a larger crown area than that of C. coggygria, which would intercept more light, reduce the temperature of the topsoil and evaporation of soil water, and eventually alleviate the negative effects of drought on $C$. coggygria when it is grown close to $R$. typhina. It has been reported that shade can alleviate the negative effects of drought on Acer buergerianum [56], which is consistent with our results. The decreased chlorophyll a to chlorophyll b ratio of $C$. coggygria under drought conditions provided further evidence to this view, because decreased chlorophyll a to chlorophyll b ratio enables the plant to obtain more light in shade [56]. Therefore, the increase in the competitive ability of $C$. coggygria over $R$. typhina under drought condition was likely caused by weaker drought effects on C. coggygria in the mixture pots compared with that in the monoculture pots. In mixture pots, the root biomass and RBR of both species remained unchanged with the increasing drought stress, and $R$. typhina had higher root biomass, RBR, and WUE than those of $C$. coggygria, indicating that the belowground advantage of $R$. typhina to obtain water and nutrients, and utilize water more effectively than $C$. coggygria was independent of soil water content.

The A and Gs (stomatal conductance) of C. coggygria simultaneously decreased by both drought and the competition with $R$. typhina, indicating the stomatal closure of $C$. coggygria in response to drought and competition. The responses of C. coggygria in the present study support the carbon-starvation hypothesis $[57,58]$, in which a plant reduces Gs as the soil water potential decreases, decreasing photosynthetic carbon uptake; thus, resulting in lower biomass compared with that under other water treatment conditions in monoculture and mixed culture. Carbohydrate reserves deplete due to continued demand for carbohydrates in order to maintain metabolism, and the plant might die due to its inability to resist attack from biotic agents or starvation, whichever occurs first [58]. The stomatal conductance and net photosynthesis rate of Acer platanoides and Fagus sylvatica were found to decrease simultaneously under drought plus competition conditions [30], which is consistent with that observed in C. coggygria in the present study. In contrast to C. $\operatorname{cog}$ gygria, $R$. typhina maintained constant $\mathrm{A}$, and $\mathrm{Gs}$ in the mixture pots, regardless of the water conditions, indicating that $R$. typhina might have adopted another mechanism to cope with drought. Some species maintain positive carbon gain under drought conditions, allowing the midday leaf water potential to decline [57, 58 ] by keeping the stoma open. In this case, $R$. typhina continued to accumulate carbohydrate under drought 
condition, maintaining the biomass constant compared with that under well-watered condition [57], and they can withstand prolonged drought condition before carbon starvation [58].

The invasive $R$. typhina and the native C. coggygria displayed differences in many growth-related parameters when they were cultivated either separately or together. This is consistent with the phenotypic divergence hypothesis [13] holding that the functional traits differences contribute to the invasion success of exotic species. Rhus typhina maintained its absolute dominance against C. coggygria in both well-watered and drought environments by possessing higher photosynthetic capacity, larger crown area, taller shoot height, and higher RGR-H, leaf and root biomass, and WUE, outcompeting C. coggygria vertically and horizontally. The growth of C. coggygria was strongly limited by the competition from $R$. typhina; however, this competition alleviated the negative effects of drought on C. coggygria mainly by providing shade environment. Drought alleviated the asymmetric competition of $R$. typhina over $C$. coggygria to some extent because drought had stronger negative effects on than on C. coggygria in the mixture pots and R. typhina is better capable of exploiting excess water resource. Our observation provides further evidence for the fluctuating resource availability theory [21]. Under drought conditions, C. coggygria suffered from carbon starvation, whereas, $R$. typhina retained the normal carbohydrate synthesis, contributing to the dominance of $R$. typhina. However, both the species would experience the risk of hydraulic failure under severe drought conditions. Our observation confirmed the robustness of the comparative trait differences between invasive and non-invasive species across environmental gradients [9].

\section{Conclusions}

Rhus typhina grows slowly at the seedling stage, but grows fast once established [59]. Therefore, $R$. typhina probably will continue its dominance when grown adjacent to C. coggygria in a long term. As the glasshouse study cannot simulate the complex natural environments, our observation should not be extrapolated arbitrarily to field conditions. Given the widely acknowledged invasiveness of $R$. typhina and the extensive use of the two species in reforestation and urban greening, field studies with closer attention on the interaction between $R$. typhina and C. coggygria in the future global change scenarios is necessary to better understand the invasion of $R$. typhina and to take appropriate precautionary and management measures. Our study contributes to a better understanding of invasive mechanisms of alien tree species such as $R$. typhina under various moisture conditions and predicts its future expansion under global climate change scenarios.

\section{Methods \\ Study site and plant materials}

The experiment was conducted in the greenhouse of Fanggan Research Station of Shandong University $\left(36^{\circ} 26^{\prime} \mathrm{N}, 117^{\circ} 27^{\prime} \mathrm{E}\right)$, which is located in the central mountainous region of Shandong Province, China. The region has a typical temperate monsoon climate, with an average annual precipitation of approximately 600 $800 \mathrm{~mm}$, most of which occurs from June to August (60-70\%), which was collected from National Climate Center of China in 2011. The predominant vegetation in this area is mixed forests of the warm temperature zone. The soil is a yellow cinnamon soil with limestone as the parent material [60].

The seeds of both species were collected from the Loahu hill $\left(36^{\circ} 43^{\prime} \mathrm{N}, 117^{\circ} 47^{\prime} \mathrm{E}\right)$ near the research station during the October of 2011.The seeds were stored at $0-4{ }^{\circ} \mathrm{C}$ during winter in the fridge $(\mathrm{BC}-50 \mathrm{ES}$, Haier Company, China). During the late April of 2012, the seeds were soaked in distilled water for $24 \mathrm{~h}$ before they were allowed to germinate in a growth chamber (PRX-1500C-LED, Tianlin Technology Co., Ltd, China). A voucher specimen of this material has been deposited in the herbarium of Shandong University. Healthy and uniformly germinated seedlings were transplanted into plastic pots ( $320 \mathrm{~mm}$ height $\times 290 \mathrm{~mm}$ diameter) with one or two seedling(s) per pot. Each pot was filled with $6 \mathrm{~kg}$ of loam and $2 \mathrm{~kg}$ of sand, which was carefully passed through a $2-\mathrm{mm}$ sieve to remove debris, and thoroughly mixed.

\section{Experimental design}

Three water treatments, 30-40\% (W1), 50-60\% (W2), and $70-80 \%$ (W3) of field capacity (FC), were applied to three plant cultivation treatments. The plant cultivation treatments included monoculture pots that contained one seedling of either species (R. typhina or $C$. coggygria) and mixture pots that contained one seedling of both the species.

The pots were randomly arranged in the greenhouse and re-randomized at regular intervals (7 days) throughout the experiment. The pots received compensatory irrigation after weighing daily at 18:00 $\mathrm{h}$ to maintain constant soil moisture level. Insects and weeds were controlled manually. Each treatment contained eight pots. The study was carried out from July 6 to September 5 of 2012, and it lasted for 62 days. 


\section{Measurement of plant traits}

The gas exchange parameters were measured on August 20 and 21, 2012, using a portable leaf gas exchange system (GFS-3000; WalzGmbH, Effeltrich, Germany). Light was supplied by a red-blue light-emitting diode set at an irradiance of $2000 \mu \mathrm{mol} \mathrm{m}{ }^{-2} \mathrm{~s}^{-1}$ (PAR) to ensure that all seedlings were light saturated. The airflow through the leaf chamber was set to $400 \mu \mathrm{mol}^{-1} \mathrm{~s}^{-1}$, chamber temperature to ambient temperature, and $\mathrm{CO}_{2}$ concentration to $400 \mu \mathrm{mol} \mathrm{mol}^{-1}(\mathrm{Ca})$. The net photosynthetic rate (A), stomata conductance (Gs), transpiration rate $(\mathrm{E})$, and intercellular $\mathrm{CO}_{2}$ concentration $(\mathrm{Ci})$ were recorded. The water use efficiency (WUE) was calculated as A/E. Three healthy and fully expanded leaves on the upper shoot of three seedlings were used to measure the parameter in each treatment.

The concentration of chlorophyll was determined on August 24 and 25, 2012, according to a previous study [61], using a UV-2100 spectrophotometer (Unico, Shanghai, China). Six healthy and fully expanded leaves on the upper shoot of three seedlings (two leaves per seedling) per treatment were collected to determine the chlorophyll concentration.

The shoot height and crown area were measured at the end of the experiment. The crown area was calculated as: crown area $=\pi \mathrm{a} b$ (where, $\mathrm{a}$ and $\mathrm{b}$ are the lengths of diagonal). Five pots were selected to measure the shoot height and crown area. The relative growth rate of shoot height (RGR-H) was calculated as: RGR-H $=(\mathrm{InH} 2-\mathrm{InH} 1) / \Delta \mathrm{t}$; where, $\mathrm{H} 2$ and $\mathrm{H} 1$ are the shoot height values measured at the end and the beginning of the treatment, and $\Delta t$ is the time duration.

Five pots per treatment were harvested thereafter, washed thoroughly with tap water, and divided into stems, leaves, and roots. The leaf area of all the plants was measured using a scanner (Epson Perfection V700; Seiko Epson, Japan) and calculated using an image analyzer (Image Pro Plus Version 4.5; Media Cybernetic, Inc., Silver Spring, MD, USA). The dry weight of various plant fractions was determined after drying in an oven for $48 \mathrm{~h}$ at $100{ }^{\circ} \mathrm{C}$. The specific area (leaf area/leaf biomass, SLA), leaf area index (leaf area/crown area, LAI), root biomass ratio (root biomass/total biomass, RBR), stem biomass ratio (stem biomass/total biomass, $\mathrm{SBR}$ ), and leaf biomass ratio (leaf biomass/total biomass, LBR) were calculated thereafter.

\section{Statistical analyses}

To evaluate the major effects and interactions of water and cultivation treatments, the two-way analysis of variance (ANOVA) was performed using plant performance and functional traits of each species. The data were log-transformed when necessary to meet the normality and homogeneity of variance assumptions of ANOVA. However, for clarity the untransformed data are presented. The two-way ANOVA and Duncan's multiple range test (DMRT) at 95\% confidence level for all plant variables were conducted.

The dominance of the target species in mixed cultures was estimated using the log response ratio (lnRR) [20, 62, 63], calculated using the following formula: $\ln R \mathrm{R}=\ln$ (total biomass of a target plant grown in monoculture/total biomass of a target plant grown in mixed culture). The species $\ln R R$ analyzed by the oneway ANOVA was expressed separately for each species.

All the statistical analyses were performed using the IBM SPSS 21.0 software package (IBM Inc., Armonk, NY, USA). Bar charts were drawn using Origin 9.0 software (OriginLab Co., Northampton, MA, USA).

\section{Abbreviations}

RGR-H: Relative growth rate of height; LAl: Leaf area index; SLA: Specific leaf area; Chl: Total chlorophyll concentration; Chla/Chlb: Chlorophyll a to b ratio; A: Maximum net photosynthetic rate; E: Transpiration rate; Gs: Stomatal conductance; Ci: Intercellular carbon dioxide concentration; WUE: Water use efficiency; FC: Field capacity; LAI: Leaf area index; RBR: Root biomass ratio; SBR: Stem biomass ratio; LBR: Leaf biomass ratio; InRR: Log response ratio.

\section{Acknowledgements}

We are grateful to Yifu Yuan, Yuan Yuan, Jikai Yang and Xiang Ma for their help during the experiment. We are grateful to Doctor Wei Li from Qingdao Agricultural University to undertake the formal identification of the plant material used in our study. Thanks to professional editors at Editage for their English editing.

\section{Authors' contributions}

$X G$ and $W G$ conceived and designed the experiments. $X R, Z X, M L$ and $X G$ carried out the experiment and data collection. XG, ZX, XR and $J L$ analyzed the data and wrote the draft manuscript. All authors contributed to the editing and revising of the final version of the manuscript. All authors read and approved the final manuscript.

\section{Funding}

This study was financially supported for the design of the study, experiment, the data collection, analysis, and writing the manuscript by National Natural Science Foundation of China $(31970347,31770361,31500264)$ and Key Research and Development Program of Shandong Province, China (No. 2019GSF109070).

\section{Availability of data and materials}

All data generated or analyzed during this study are included in this published article. The datasets in this study are available from the corresponding author on reasonable request.

Ethics approval and consent to participate

Not applicable.

Consent for publication

Not applicable.

Competing interests

The authors declare that they have no competing interests.

Author details

${ }^{1}$ College of Landscape Architecture and Forestry, Qingdao Agricultural University, Qingdao 266109, People's Republic of China. ${ }^{2}$ Institute of Ecology 
and Biodiversity, College of Life Sciences, Shandong University, Qingdao 266237, People's Republic of China. ${ }^{3}$ Shandong Provincial Engineering and Technology Research Center for Vegetation Ecology, Shandong University, Qingdao 266237, People's Republic of China. ${ }^{4}$ Institute of Environmental Research, Shandong University, Qingdao 266237, People's Republic of China.

Received: 31 July 2019 Accepted: 7 March 2020

Published online: 30 March 2020

\section{References}

1. IPCC: Climate change 2014: synthesis report. Contribution of Working Groups I, II and III to the Fifth Assessment Report of the Intergovernmental Panel on Climate Change. In: 2014.

2. Shi P, Preisler HK, Quinn BK, Zhao J, Huang W, Röll A, Cheng X, Li H, Hölscher D. Precipitation is the most crucial factor determining the distribution of moso bamboo in Mainland China. Glob Ecol Conserv. 2020;22:e00924.

3. Ciais P, Reichstein M, Viovy N, Granier A, Ogée J, Allard V, Aubinet M, Buchmann N, Bernhofer C, Carrara A. Europe-wide reduction in primary productivity caused by the heat and drought in 2003. Nature. 2005;437(7058):529.

4. Granier A, Reichstein M, Bréda N, Janssens IA, Falge E, Ciais P, Grünwald T, Aubinet M, Berbigier P, Bernhofer C. Evidence for soil water control on carbon and water dynamics in European forests during the extremely dry year: 2003. Agric For Meteorol. 2007;143(1):123-45.

5. Mariotte P, Robroek BJM, Jassey VEJ, Buttler A. Subordinate plants mitigate drought effects on soil ecosystem processes by stimulating fungi. Funct Ecol. 2015;29(12):1578-86.

6. Vila M, Espinar JL, Hejda M, Hulme PE, Jarosik V, Maron JL, Pergl J, Schaffner U, Sun Y, Pysek P. Ecological impacts of invasive alien plants: a metaanalysis of their effects on species, communities and ecosystems. Ecol Lett. 2011;14(7):702-8.

7. Isbell F, Craven D, Connolly J, Loreau M, Schmid B, Beierkuhnlein C, Bezemer TM, Bonin CL, Bruelheide H, De Luca E. Biodiversity increases the resistance of ecosystem productivity to climate extremes. Nature. 2015;526(7574):574-7

8. Landi P, Minoarivelo HO, Brännström A, Hui C, Dieckmann U. Complexity and stability of ecological networks: a review of the theory. Popul Ecol. 2018;60(4):319-45.

9. van Kleunen M, Weber E, Fischer M. A meta-analysis of trait differences between invasive and non-invasive plant species. Ecol Lett. 2010;13(2):235-45.

10. van Kleunen M, Dawson W, Schlaepfer D, Jeschke JM, Fischer M. Are invaders different? A conceptual framework of comparative approaches for assessing determinants of invasiveness. Ecol Lett. 2010;13(8):947-58.

11. Davidson AM, Jennions MD, Nicotra AB. Do invasive species show higher phenotypic plasticity than native species and if so, is it adaptive? A metaanalysis. Ecol Lett. 2011;14(4):419-31.

12. Hui C, Richardson DM, Landi P, Minoarivelo HO, Garnas J, Roy HE. Defining invasiveness and invasibility in ecological networks. Biol Invasions. 2016:18(4):971-83.

13. Ordonez A, Wright IJ, Olff H. Functional differences between native and alien species: a global-scale comparison. Funct Ecol. 2010;24(6):1353-61.

14. Tan X, Guo X, Guo W, Liu S, Du N. Invasive Rhus typhina invests more in height growth and traits associated with light acquisition than do native and non-invasive alien shrub species. Trees. 2018;32(4):1-10.

15. Duncan RP, Williams PA. Ecology-Darwin's naturalization hypothesis challenged. Nature. 2002;417(6889):608.

16. Thompson K, Hodgson JG, Rich TCG. Native and alien invasive plants: more of the same? Ecography. 1995;18(4):390-402.

17. Daehler CC. Performance comparisons of co-occurring native and alien invasive plants: implications for conservation and restoration. Annu Rev Ecol Evol Syst. 2003;34:183-211

18. Drenovsky RE, James JJ. Trait convergence and plasticity among native and invasive species in resource-poor environments. Am J Bot. 2012:99(4):629-39.

19. Scharfy D, Funk A, Venterink HO, Gusewell S. Invasive forbs differ functionally from native graminoids, but are similar to native forbs. New Phytol. 2011;189(3):818-28.
20. Dostál P. Plant competitive interactions and invasiveness: searching for the effects of phylogenetic relatedness and origin on competition intensity. Am Nat. 2011;177(5):655.

21. Davis MA, Grime JP, Thompson K. Fluctuating resources in plant communities: a general theory of invasibility. J Ecol. 2000;88:528-34.

22. Funk JL, Vitousek PM. Resource-use efficiency and plant invasion in lowresource systems. Nature. 2007;446(7139):1079.

23. Dick JTA, Alexander ME, Ricciardi A, Laverty C, Downey PO, Xu M, Jeschke JM, Saul W-C, Hill MP, Wasserman R, et al. Functional responses can unify invasion ecology. Biol Invasions. 2017;19(5):1667-72.

24. Vonesh J, McCoy M, Altwegg R, Landi P, Measey J. Functional responses can't unify invasion ecology. Biol Invasions. 2017;19(5):1673-6.

25. Dick JTA, Alexander ME, Ricciardi A, Laverty C, Downey PO, Xu M, Jeschke JM, Saul W-C, Hill MP, Wasserman R, et al. Fictional responses from Vonesh et al. Biol Invasions. 2017;19(5):1677-8.

26. Vonesh J, McCoy M, Altwegg R, Landi P, Measey J. Rather than unifying invasion biology, Dick et al's approach rests on subjective foundations. Biol Invasions. 2017;19(5):1679-80.

27. Yu X, Hui C, Sandhu HS, Lin Z, Shi P. Scaling relationships between leaf shape and area of 12 Rosaceae species. Symmetry. 2019;11(10):1255.

28. Bradford MA, Schumacher HB, Sebastian C, Till E, Newingtion JE, Tordoff GM. Impacts of invasive plant species on riparian plant assemblages: interactions with elevated atmospheric carbon dioxide and nitrogen deposition. Oecologia. 2007;152(4):791-803.

29. Luo Y, Guo W, Yuan Y, Liu J, Du N, Wang R. Increased nitrogen deposition alleviated the competitive effects of the introduced invasive plant Robinia pseudoacacia on the native tree Quercus acutissima. Plant Soil. 2014;385(1-2):63-75

30. Hommel R, Siegwolf R, Zavadlav S, Arend M, Schaub M, Galiano L, Haeni M, Kayler ZE, Gessler A. Impact of interspecific competition and drought on the allocation of new assimilates in trees. Plant Biol. 2016;18:785-96.

31. Zou J, Rogers WE, Siemann E. Plasticity of Sapium sebiferum seedling growth to light and water resources: Inter- and intraspecific comparisons. Basic Appl Ecol. 2009;10(1):79-88.

32. Huang W, Ratkowsky D, Hui C, Wang P, Su J, Shi P. Leaf fresh weight versus dry weight: which is better for describing the scaling relationship between leaf biomass and leaf area for broad-leaved plants? Forests. 2019;10(3):256.

33. Castillo JM, Leira-Doce P, Carrión-Tacuri J, Muñoz-Guacho E, Arroyo-Solís A, Curado G, Doblas D, Rubio-Casal AE, Álvarez-López AA, RedondoGómez S, et al. Contrasting strategies to cope with drought by invasive and endemic species of Lantana in Galapagos. Biodivers Conserv. 2006;16(7):2123-36

34. te Beest M, Elschot K, Olff H, Etienne RS. Invasion success in a marginal habitat: an experimental test of competitive ability and drought tolerance in Chromolaena odorata. PLoS ONE. 2013;8(8):e68274.

35. Du N, Tan X, Li Q, Liu X, Zhang W, Wang R, Liu J, Guo W. Dominance of an alien shrub Rhus typhina over a native shrub Vitex negundo var. heterophylla under variable water supply patterns. PLOS ONE. 2017;12(4):e0176491.

36. Yuan Y, Guo W, Ding W, Du N, Luo Y, Liu J, Xu F, Wang R. Competitive interaction between the exotic plant Rhus typhina $\mathrm{L}$. and the native tree Quercus acutissima Carr. in Northern China under different soil N:P ratios. Plant Soil. 2013;372(1-2):389-400.

37. Weber $E_{1}$ Gut $D$. Assessing the risk of potentially invasive plant species in central Europe. J Nat Conserv. 2004;12(3):171-9.

38. Ewald W, Sun SG, Bo L. Invasive alien plants in China: diversity and ecological insights. Biol Invasions. 2008;10(8):1411-29.

39. Wang G, Jiang G, Yu S, Li Y, Liu H. Invasion possibility and potential effects of Rhus typhina on Beijing municipality. J Integr Plant Biol. 2008;50(5):522-30.

40. Wang W, Tian CY, Li YH, Li Y. Molecular data and ecological niche modelling reveal the phylogeographic pattern of Cotinus coggygria (Anacardiaceae) in China's warm-temperate zone. Plant Biol. 2014;16(6):1114-20.

41. Lei Y-K, Wang W, Liu Y-P, He D, Li Y. Adaptive genetic variation in the smoke tree (Cotinus coggygria Scop.) is driven by precipitation. Biochem Syst Ecol. 2015;59:63-9.

42. Wang W, Li Z, Li Y. Isolation and Characterization of Microsatellite Markers for Cotinus coggygria Scop. (Anacardiaceae) by 454 pyrosequencing. Molecules. 2014;19(3):3813. 
43. Byun C, De Blois S, Brisson J. Plant functional group identity and diversity determine biotic resistance to invasion by an exotic grass. J Ecol. 2013;101(1):128-39.

44. Knochel DG, Flagg C, Seastedt TR. Effects of plant competition, seed predation, and nutrient limitation on seedling survivorship of spotted knapweed (Centaurea stoebe). Biol Invasions. 2010;12(11):3771-84

45. Vila M, Gomez AM, Maron JL. Are alien plants more competitive than their native conspecifics? A test using Hypericum perforatum L. Oecologia. 2003:137(2):211-5.

46. Mangla S, Sheley RL, James JJ, Radosevich SR. Intra and interspecific competition among invasive and native species during early stages of plant growth. Plant Ecol. 2011;212(4):531-42.

47. Pyšek P, Richardson DM. Traits associated with invasiveness in alien plants: where do we stand? In: Nentwig W, editor. Biological invasions. Berlin: Springer; 2007. p. 97-125.

48. te Beest M, Stevens N, Olff H, van der Putten WH. Plant-soil feedback induces shifts in biomass allocation in the invasive plant Chromolaena odorata. J Ecol. 2009;97(6):1281-90.

49. Hierro JL, Maron JL, Callaway RM. A biogeographical approach to plant invasions: the importance of studying exotics in their introduced and native range. J Ecol. 2005;93(1):5-15.

50. Crous CJ, Jacobs SM, Esler KJ. Wood anatomical traits as a measure of plant responses to water availability: invasive Acacia mearnsii De Wild. compared with native tree species in fynbos riparian ecotones, South Africa. Trees Struct Funct. 2012;26(5):1527-36.

51. Jimenez MA, Jaksic FM, Armesto JJ, Gaxiola A, Meserve PL, Kelt DA, Gutierrez JR. Extreme climatic events change the dynamics and invasibility of semi-arid annual plant communities. Ecol Lett. 2011;14(12):1227-35.

52. Coomes DA, Lines ER, Allen RB. Moving on from Metabolic Scaling Theory: hierarchical models of tree growth and asymmetric competition for light. J Ecol. 2011;99(3):748-56.

53. Pretzsch H, Biber P. Size-symmetric versus size-asymmetric competition and growth partitioning among trees in forest stands along an ecological gradient in central Europe. Can J For Res. 2010;40(2):370-84.

54. Falster DS, Westoby M. Plant height and evolutionary games. Trends Ecol Evol. 2003;18(7):337-43.
55. Lamarque LJ, Delzon S, Lortie CJ. Tree invasions: a comparative test of the dominant hypotheses and functional traits. Biol Invasions. 2011;13(9):1969-89.

56. Guo X, Guo W, Luo Y, Tan X, Du N, Wang R. Morphological and biomass characteristic acclimation of trident maple (Acer buergerianum Miq.) in response to light and water stress. Acta Physiologiae Plantarum. 2013;35(4):1149-59.

57. Blackman CJ, Brodribb TJ, Jordan GJ. Leaf hydraulics and drought stress: response, recovery and survivorship in four woody temperate plant species. Plant Cell Environ. 2009;32(11):1584-95.

58. McDowell N, Pockman WT, Allen CD, Breshears DD, Cobb N, Kolb T, Plaut J, Sperry J, West A, Williams DG, et al. Mechanisms of plant survival and mortality during drought: why do some plants survive while others succumb to drought? New Phytol. 2008;178(4):719-39.

59. Zhang Z, Jiang C, Zhang J, Zhang H, Shi L. Ecophysiological evaluation of the potential invasiveness of Rhus typhina in its non-native habitats. Tree Physiol. 2009;29(11):1307-16.

60. Wang RQ, Zhou GY. The Vegetation of Shandong Province. Jinan: Shandong Science and Technology Publisher; 2000.

61. Lichtenthaler HK, Wellburn AR. Determinations of total carotenoids and chlorophylls $\mathrm{a}$ and b of leaf extracts in different solvents. Biochem Soc T. $1983 ; 11: 591-2$

62. Goldberg DE, Rajaniemi T, Gurevitch J, Stewart-Oaten A. Empirical approaches to quantifying interaction intensity competition and facilitation along productivity gradients. Ecology. 1999;80(4):1118-31.

63. Weigelt A, Steinlein T, Beyschlag W. Does plant competition intensity rather depend on biomass or on species identity? Basic Appl Ecol. 2002;3(1):85-94.

\section{Publisher's Note}

Springer Nature remains neutral with regard to jurisdictional claims in published maps and institutional affiliations.
Ready to submit your research? Choose BMC and benefit from:

- fast, convenient online submission

- thorough peer review by experienced researchers in your field

- rapid publication on acceptance

- support for research data, including large and complex data types

- gold Open Access which fosters wider collaboration and increased citations

- maximum visibility for your research: over $100 \mathrm{M}$ website views per year

At BMC, research is always in progress.

Learn more biomedcentral.com/submissions 